\title{
Analyse des systèmes d'information et intégration des données spatialisées
}

\author{
par Alain Vidal \\ Cemagref, Division Irrigation
}

Solène Laloux

Compagnie d'aménagement des côteaux de Gascogne

Damien Lepoutre

GEOSYS

\section{INTRODUCTION}

Depuis quelques années, la généralisation d'outils informatiques matériels et logiciels standardisés et relativement peu coûteux a permis le développement de systèmes d'information pour la gestion de l'irrigation et du drainage dans un certain nombre de pays. Ce développement a souvent porté ses fruits, mais on constate parfois que les systemes d'information mis en place sont sous-utilisés, sont devenus obsolètes, ou encore ne permettent pas l'intégration de nouvelles fonctionnalités. Ce constat résulte souvent de la démarche adoptée pour mettre en place un tel système, qui est souvent plus orientée vers l'outil que vers les besoins de l'utilisateur. En effet, s'il est largement admis que la gestion des ressources doit être globale, sa mise en œuvre est délicate, car elle implique une diversité d'acteurs dont les objectifs et les actions peuvent diverger, voire se concurrencer, en particulier lorsque les ressources sont limitées. De fait, les gestionnaires souhaitent disposer à la fois d'informations très hétérogènes (qualitatives, quantitatives, administratives,...), frables et spatialisées, et dans la plupart des cas, il est nécessaire d'analyser l'ensemble des processus de gestion avant la mise en place d'un système d'information adapté.

\section{Analysis of information systems and integration of spatially-distributed data}

It is usually admitted that resources management has to be global. However implementing such a management is not easy, as it implies various parthers, whose objectives and actions mov be dwergent, even competitive, especially when resources are limited. Managers now days require heterogeneous (qualitative, quantitative, administrative...), reliable and spatialty distributed information. Two technologies todav available can here be potentially used. remote sensing and geographie information systems (GS). Therefore. in the frame of the ICID Working Group on Decision Systems for Land and Water Management, AFEDD (the French National Committee of ICD) constituted in 1994 a Working Group on Remote Sensing and GIS, whose objectives are, anong orhers, the preparation of two $1 \mathrm{CW}$ guiles, on the use of remote sensing and GIS in irrigation and draingse.

The originalit, of the presented approach. to be further used for the nanagement of irrigation andlor drat nage systems, results trom the application of information sistems analysis usually used in company audi ting. This analysis has been adapted to specific constraints of geographic information, based on numerous experiences in councelling and in development of dectsion support systens for public authonities managing large territories. 
De plus en plus souvent, la télédétection et les systèmes d'information géographique (SIG) sont des composantes de tels systèmes d'information, et leur intégration est particulièrement délicate, car elles permettent de disposer et de manipuler des données spatialisées. C'est pourquoi, dans le cadre du groupe de travail CIID sur les systèmes d'aide à la décision pour la gestion des terres et des eaux, l'AFEID (Association Française pour l'Etude des Irrigations et du Drainage) s'est dotée en 1994 d'un groupe de travail sur la télédétection et les SIG, dont les objectifs sont notamment la réalisation de deux guides CIID, sur l'utilisation de la télédétection et des SIG en irrigation et drainage.

La préparation de ces guides nous a amenés à considérer l'ensemble de la démarche nécessaire à la mise en place d'un système d'information pour la gestion de l'irrigation et du drainage, pour aboutir à des recommandations techniques et pratiques favorisant l'intégration d'informations spatialisées dans de tels systèmes. Nous présentons ici :

1. la démarche qui a été adoptée :

2. comment, sur un cas concret de gestion de la ressource en eau utilisant la télédétection et un SIG, cette démarche a été mise en œuvre ;

3. comment cette démarche pourrait être utilisée dans le cas d'un périmètre irrigué, sachant que les travaux menés par le groupe de travail ne sont pas entièrement achevés.

\section{MÉTHODE}

L'originalité de la démarche choisie, à l'usage de gestionnaires de périmètres irrigués et/ou drainés, découle de l'application de l'analyse des systèmes d'informations plus classiquement utilisée en audit d'entreprise, et présentée à la figure I (Le cheminement logique consiste à partir de la base du triangle (stratégie), remonter sur la gauche pour l'analyse et redescendre par la droite pour la mise en ouvre du système d'information. Dans un certain nombre de cas, la partie ascendante est négligée, et on cherche simplement à organiser des données disponibles, par exemple des images satellitaires, des enquêtes, des relevés etc. pour mettre en ouvre le système, ce qui conduit le plus souvent à un échec.).

Cette analyse a été adaptée aux contraintes spécifiques de la composante géographique de l'information, sur la base de nombreuses expériences de conseil et de mise en place de systèmes d'aide à la décision pour les gestionnaires publics de grands territoires.

Cette démarche suit les étapes suivantes

- analyse de la stratégie du gestionnaire :

- détermination des indicateurs et des facteurs de succès et d'échec:

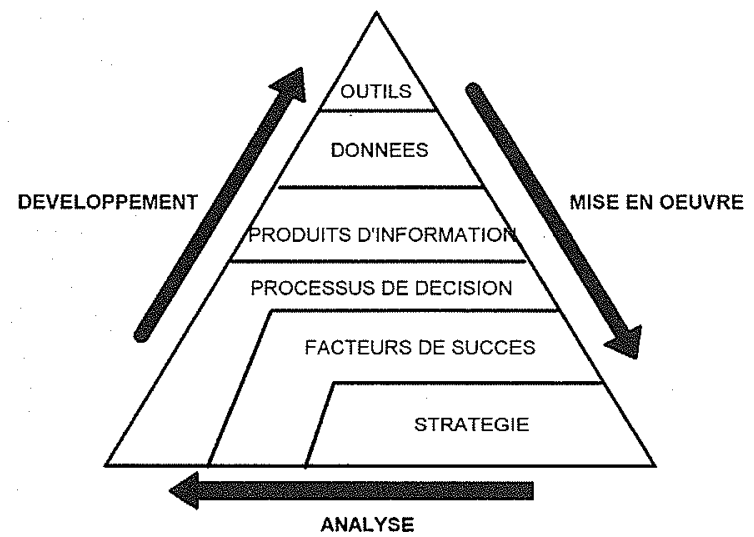

1. Représentation de l'analyse des systèmes d'information appliquée à I'information géographique.
- analyse des processus de décision interne, incluant la description du système d'information auxquels ces processus font appel ;

- définition des produits d'information, classés en cinq catégories selon le délai d'utilisation :

1) produits d'information-communication,

2) produits permettant des décisions stratégiques (objectifs à long et moyen terme, planification),

3) tactiques (gestion à moyen terme),

4) logistiques (gestion à court terme, opération et maintenance), et

5) produits d'alerte

- analyse de l'utilisation de ces produits d'information ;

- connaissance des données, normes et contraintes pour élaborer ces produits ;

- analyse des outils à mettre en ouvre pour disposer des produits d'information: c'est à cette étape seulement que seront abordés la télédétection et les SIG.

Cette méthode a été appliquée dans un cas de la gestion des ressources en eau sur le Bassin de la Seine (France), qui est présenté dans la deuxième partie.

Par ailleurs, dans le cadre du groupe de travail AFEID sur la télédétection et les SIG, c'est la grille d'analyse des processus de décision et des fonctions utilisées par une Société d'Aménagement Régional, la SCP (Société du Canal de Provence) qui a été prise comme base de travail. La confrontation de l'expérience d'organismes variés tels que centres de recherche, compagnies d'aménagement, sociétés de services (en France et à l'étranger) a ensuite enrichi l'analyse des métiers liés à l'irrigation et des produits d'information qu'ils engendrent. Les résultats, provisoires, de cette analyse, présentés dans la troisième partie de ce rapport, sont centrés sur un nombre limité de produits d'information, les plus pertinents en réponse aux attentes des gestionnaires.

\section{UN EXEMPLE D'APPLICATION EN GESTION DES RESSOURCES EN EAU}

Cet exemple concerne la mise en place d'un système d'aide à la décision pour l'extension des ressources du Bassin de la Seine (France) en fonction des futurs besoins en eau. Cette étude associe deux utilisateurs, l'Agence de l'Eau SeineNormandie (AESN) et l'Institution Interdépartementale des Barrages Réservoirs du bassin de la Seine (IIBRBS), et deux opérateurs représentés au sein du groupe de travail AFEID, la Compagnie d'Aménagement des Coteaux de Gascogne (CACG) et la société GEOSYS.

\subsection{La demande}

Afin d'anticiper sur les besoins futurs en eau dans le bassin amont de la Seine, à l'horizon 2015, l'AESN et l'IIBRBS souhaitent disposer d'informations sur les besoins qualitatifs et quantitatifs afin d'avoir en main les éléments décisionnels pour garantir les ressources en eau futures. La demande d'informations concerne donc autant l'existant que le futur. Ces dernières informations doivent reposer sur des hypothèses selon trois scénarios d'évolution des besoins et des actions à mener :

- évolution tendancielle,

- évolution contrôlée,

- évolution maîtrisée.

\subsection{Analyse de la stratégie des acteurs}

L'AESN et l'IIBRBS sont des organismes «techniques » devant apporter des éléments «incontestables» et «objec- 
tifs », pour aider les décideurs à définir des actions garantissant la quantité et la qualité des ressources en eau de la région parisienne et du bassin amont de la Seine. Les informations à fournir sont donc de type «stratégique ». De celles-ci dépendront les décisions d'investissements très lourds. Elles doivent donc être fỉables, plutôt que précises. Or étant appuyées sur des prévisions, elles sont par nature hypothétiques. La décision stratégique se devait donc de prendre en compte les risques sur les hypothèses afin d'évaluer les enjeux et risques sur chaque décision qui en découle.

\subsection{Indicateurs et facteurs clés de succès}

L'analyse des facteurs clés de succès de l'étude a permis de constater qu'il était important que les décideurs ne puissent remettre en cause les informations à la base du travail, tout en connaissant les hypothèses et risques. Les actions et les engagements qui découlent de l'étude sont nombreux et étalés dans le temps (jusqu'en 2015). La possibilité de remplacer des données prévisionnelles par la réalité au fur et à mesure du temps était aussi un facteur clé de succès. Il fallait donc pérenniser l'expertise acquise (données collectées, traitements et modèles appliqués). L'évolution dans le temps peut aussi amener à revoir des scénarios avec de nouvelles hypothèses pour le futur. En résumé, les facteurs clés du succès identifiés sont :

- la disponibilité d'informations objectives (informations reconnues par tous les acteurs).

- la non remise en cause des informations de base,

- la possibilité de « rejouer» des scénarios pour des paramètres ou données mises à jour,

- la facilité d'utilisation et de consultation (convivialité),

- la pérennité de l'expertise acquise (données collectées)

\subsection{Processus de décision}

Il s'agit ici d'aider à la décision sur des éléments fiables, pour planifier des-actions ou des structures améliorant I'accès aux ressources en eau. La maîtrise des risques sur les hypothèses implique de conduire des simulations en modifiant, si possible interactivement, les hypothèses pour le futur. Il ne s'agit donc pas de réaliser une étude avec un résultat pour chacune des trois hypothèses, mais bien de mettre en place un système d'information très convivial et interactif, permettant d'analyser l'impact sur les résultats d'un intervalle de confiance sur ces hypothèses.

Les décisions et évolutions sur le bassin modifieront les données qui remplaceront au fur et à mesure les hypothèses de simulation. Le processus de décision s'appuiera donc sur une base de données très structurée permettant de réaliser de nouvelles simulations en mettant à jour des données. Le système d'information s'appuie totalement sur une base de données relationnelles. L'évolution des connaissances et expertises amènera à modifier et optimiser les critères de décision, ainsi que les modèles de simulation. Les décideurs feront donc évoluer le système de simulation. Le système doit par conséquent être modulaire et documenté.

L'ensemble des informations mises en œuvre étant géographiques et interdépendantes, le système devait donc être un système d'information géographique.

\subsection{Produits d'information}

Les produits d'informations sont «actifs », et se composent de :

- une base d'informations quantitatives (besoins),

- une modélisation (prospective)

De ce fait, il s'agit d'un système de consultation et d'un système de simulation. Le caractère spatial des entités mises en œuvre induit des actions de géo-consultation et de géosimulation. Les clés de sélection sont géographiques (bassins versants, communes, départements).

\section{解 3.6 Informations de base}

Les regroupements d'informations selon plusieurs critères thématiques (administratifs, hydrographiques, gestion, infrastructures, etc.) rendent difficiles la constitution d'un objet élémentaire commun. La commune constitue une maille élémentaire de référence. En effet, elle permet une cohérence géographique et thématique tout en garantissant une représentativité des données associées (occupation du sol, données socio-économiques, consommations etc.). Au niveau d'analyse choisi (grand bassin versant), la maille " communale $»$ permet de consolider les informations par:

- entités administratives (département, région),

- entités de gestion (distributeurs),

- entités d'infrastructures (réseau interconnectés),

Tableau 1. - Demande des utilisateurs, analyse et solutions retenues pour la gestion prévisionnelle des ressources sur le bassin de la Seine, France.

\begin{tabular}{|l|l|l|l|}
\hline Système de décision & \multicolumn{1}{|c|}{ Demande exprimée } & \multicolumn{1}{|c|}{ Analyse } & \multicolumn{1}{|c|}{ Solution mise en œuvre } \\
\hline & $\begin{array}{l}\text { Evaluer les enjeux et } \\
\text { risques d'une hypothèse } \\
\text { d'évolution des besoins } \\
\text { en eau par rapport aux } \\
\text { ressources }\end{array}$ & $\begin{array}{l}\text { Disposer d'informations } \\
\text { de base fiables }\end{array}$ & $\begin{array}{l}\text { Système d'aide à la décision } \\
\text { basé sur une simulation } \\
\text { paramétrée et une base de } \\
\text { données fiable et cohérente }\end{array}$ \\
\hline Produits d'information & $\begin{array}{l}\text { Disposer de résultats } \\
\text { par rapport à des scéna- } \\
\text { rios établis }\end{array}$ & $\begin{array}{l}\text { Evaluer l'impact des } \\
\text { scénarios choisis }\end{array}$ & $\begin{array}{l}\text { Permettre de modifier et d'ajuster } \\
\text { les paramètres pris pour chaque } \\
\text { scénario }\end{array}$ \\
\hline Données de base & $\begin{array}{l}\text { Hypothèses d'évolution } \\
\text { simples } \\
\text { Base d'informations } \\
\text { fiables et non remises } \\
\text { en cause }\end{array}$ & $\begin{array}{l}\text { Structuration des infor- } \\
\text { mations et validation de } \\
\text { ces dernières }\end{array}$ & $\begin{array}{l}\text { Base de données relationnelles } \\
\text { associées à des entités géogra- } \\
\text { phiques } \\
\text { Structuration des hypothèses }\end{array}$ \\
\hline Outils & $\begin{array}{l}\text { Expertise } \\
\text { Modularité }\end{array}$ & $\begin{array}{l}\text { Ergonomie } \\
\text { Simplicité de mise en } \\
\text { Mive à jour des données } \\
\text { Mise ànes }\end{array}$ & $\begin{array}{l}\text { Outil totalement dédié construit } \\
\text { sur SlG - Interfaces conviviales }\end{array}$ \\
\hline
\end{tabular}


Tableau 2. - Analyse des processus de décision et des fonctions stratégiques dans un système irrigué/drainé, définition des produits d'information et de leurs caractéristiques.

\begin{tabular}{|c|c|c|c|c|c|}
\hline $\begin{array}{l}\text { Critères } \\
\text { Missions }\end{array}$ & $\begin{array}{l}\text { Description } \\
\text { des fonctions }\end{array}$ & $\begin{array}{c}\text { Produits } \\
\text { d'informations }\end{array}$ & $\begin{array}{c}\text { Nature } \\
\text { spatialisée }\end{array}$ & $\begin{array}{c}\text { Echelle } \\
\text { spatiale (1) }\end{array}$ & $\begin{array}{c}\text { Caractéristique } \\
\text { temporelle }\end{array}$ \\
\hline $\begin{array}{l}\text { Planification } \\
\text { de la mainte- } \\
\text { nance }\end{array}$ & $\begin{array}{l}\text { Fixer les objectifs de } \\
\text { maintenance. Analyser } \\
\text { les indicateurs de } \\
\text { niveau de maintenance } \\
\text { réalisée, fixer les } \\
\text { orientations de budget } \\
\text { de maintenance et les } \\
\text { règles de gestion de } \\
\text { maintenance }\end{array}$ & Tableau de bord & $\begin{array}{l}\text { Non, pas } \\
\text { en straté- } \\
\text { gique }\end{array}$ & $G, S, R$ & $\begin{array}{l}\text { Horizon }>1 \text { an } \\
\text { Période }=1 \text { an }\end{array}$ \\
\hline $\begin{array}{l}\text { Planification } \\
\text { de I'exploita- } \\
\text { tion }\end{array}$ & $\begin{array}{l}\text { Définition du budget, } \\
\text { des règles de gestion } \\
\text { de l'exploitation et du } \\
\text { contrôle des ouvrages }\end{array}$ & $\begin{array}{l}\text { Indicateurs de perfor- } \\
\text { mances }\end{array}$ & Non & $G, S, R$ & $\begin{array}{l}\text { Horizon }>1 \text { an } \\
\text { Période }=1 \text { an }\end{array}$ \\
\hline $\begin{array}{l}\text { Planification } \\
\text { budgétaire }\end{array}$ & $\begin{array}{l}\text { Analyser les recettes, } \\
\text { les consommations, } \\
\text { les coúts de produc- } \\
\text { tion énergétiques et } \\
\text { leur évolution dans le } \\
\text { temps }\end{array}$ & $\begin{array}{l}\text { Prix des services, type } \\
\text { de contrat, consomma- } \\
\text { tions et surfaces }\end{array}$ & Non & $\mathrm{G}, \mathrm{S}, \mathrm{R}$ & $\begin{array}{l}\text { Horizon }>1 \text { an } \\
\text { Période }=1 \text { an }\end{array}$ \\
\hline $\begin{array}{l}\text { Evaluation de } \\
\text { la gestion } \\
\text { technique }\end{array}$ & $\begin{array}{l}\text { Analyser les indica- } \\
\text { teurs de performances } \\
\text { et de saturation des } \\
\text { réseaux et leur évolu- } \\
\text { tion dans le temps }\end{array}$ & $\begin{array}{l}\text { Indicateurs de perfor- } \\
\text { mances (graphes) }\end{array}$ & $\begin{array}{l}\text { Oui, pour } \\
\text { l'analyse }\end{array}$ & $G, S, R$ & $\begin{array}{l}\text { Horizon }>1 \text { an } \\
\text { Période }=1 \text { an }\end{array}$ \\
\hline $\begin{array}{l}\text { Planification } \\
\text { des actions } \\
\text { commerciales } \\
\text { et des exten- } \\
\text { sions }\end{array}$ & $\begin{array}{l}\text { Analyser les indica- } \\
\text { teurs d'évolution de la } \\
\text { clientèle existante, pla- } \\
\text { nifier l'action commer- } \\
\text { ciale en conséquence } \\
\text { et en fonction des } \\
\text { objectifs généraux de } \\
\text { l'organisme. Analyser } \\
\text { la demande extérieure }\end{array}$ & $\begin{array}{l}\text { Consommations, } \\
\text { enquêtes de satisfac- } \\
\text { tion, analyse des } \\
\text { besoins, analyse de } \\
\text { l'existant et des poten- } \\
\text { tialités }\end{array}$ & $\begin{array}{l}\text { Oui }=\text { géo- } \\
\text { marketing }\end{array}$ & $\mathrm{G}, \mathrm{S}, \mathrm{R}$ & $\begin{array}{l}\text { Horizon }>1 \text { an } \\
\text { Période }=1 \text { an }\end{array}$ \\
\hline $\begin{array}{l}\text { Planification } \\
\text { de l'accès aux } \\
\text { ressources }\end{array}$ & $\begin{array}{l}\text { Planifier l'extension des } \\
\text { ressources en fonction } \\
\text { des besoins et des } \\
\text { moyens actuels et futurs }\end{array}$ & $\begin{array}{l}\text { Connaissance des } \\
\text { besoins et des res- } \\
\text { sources (nappes, } \\
\text { climat, rivières) }\end{array}$ & Oui & $G, S, R$ & $\begin{array}{l}\text { Horizon }>1 \text { an } \\
\text { Période }=1 \text { an }\end{array}$ \\
\hline Qualité & & $\begin{array}{l}\text { Organisation, docu- } \\
\text { mentation, procédures }\end{array}$ & Non & $G, S, R$ & $\begin{array}{l}\text { Horizon }>1 \text { an } \\
\text { Période }=1 \text { an }\end{array}$ \\
\hline $\begin{array}{l}\text { Etablissement } \\
\text { des principes } \\
\text { légaux }\end{array}$ & $\begin{array}{l}\text { Lois sur l'eau, grands } \\
\text { principes légaux, accès } \\
\text { au foncier }\end{array}$ & Lois, décrets, règles & Non & $G, S, R$ & $\begin{array}{l}\text { Horizon }>1 \text { an } \\
\text { Période }=1 \text { an }\end{array}$ \\
\hline
\end{tabular}

(1) $\mathrm{G}=$ Global, $\mathrm{S}=$ Secteur, $\mathrm{R}=$ Réseau, $\mathrm{A}=$ Antenne, $\mathrm{B}=$ Borne.

- entités de programmation (syndicat d'études et de prospectives),

- entités hydrographiques (bassin versant),

- entités homogènes par rapport à des «types de consommation $»$.

La maille communale permet de garder une cohérence géographique entre la région Ile-de-France et le haut bassin de la Seine, tout en ayant deux niveaux de description des données thématiques associées. L'ensemble représente:

- pour l'lle-de-France : 1300 communes (8 départements), - pour le haut bassin de la Seine : 4013 communes (12 départements).

Les informations sont fiables, mais peu précises géographiquement. C'est la caractéristique principale des informations mises en œuvre pour la prise de décision.

\subsection{Utilisation de ces produits}

La géo-consultation : il s'agit d'une consultation conviviale des informations rassemblées dans le cadre de l'étude. La consultation s'effectue en désignant des éléments géographiques d'intérêt.

La géo-simulation : l'utilisation doit permettre de simuler des scénarios en paramétrant des éléments géographiques.

\subsection{Connaissance des données pour élaborer les pro-} duits

Les données de base doivent être fiables, complètes et cohérentes. 
Tableau 3. - Analyse des processus de décision et des fonctions logistiques dans un système irrigué/drainé, définition des produits d'information et de leurs caractéristiques.

\begin{tabular}{|c|c|c|c|c|c|}
\hline $\begin{array}{l}\text { Critères } \\
\text { Missions }\end{array}$ & $\begin{array}{l}\text { Description } \\
\text { des fonctions }\end{array}$ & $\begin{array}{c}\text { Produits } \\
\text { d'informations }\end{array}$ & $\begin{array}{c}\text { Nature } \\
\text { spatialisée }\end{array}$ & $\begin{array}{l}\text { Echelle } \\
\text { spatiale }\end{array}$ & $\begin{array}{l}\text { Caractéristique } \\
\text { temporelle }\end{array}$ \\
\hline $\begin{array}{c}\text { Interventions } \\
\text { de mainte- } \\
\text { nance }\end{array}$ & $\begin{array}{l}\text { Exécuter et rapporter } \\
\text { les interventions de } \\
\text { maintenance préven- } \\
\text { tive et corrective }\end{array}$ & $\begin{array}{l}\text { Tableaux de relevés } \\
\text { d'intervention }^{\prime}\end{array}$ & Oui & $\mathrm{R}, \mathrm{A}, \mathrm{B}$ & Temps réel \\
\hline $\begin{array}{l}\text { Gestion du } \\
\text { réseau }\end{array}$ & $\begin{array}{l}\text { Assurer la distribution } \\
\text { grâce aux informations } \\
\text { issues des systèmes } \\
\text { d'irrigation, gérer les } \\
\text { réseaux (automatique } \\
\text { ou manuel), archiver } \\
\text { les changements d'état } \\
\text { et les mesures, identi- } \\
\text { fier les défauts de } \\
\text { fonctionnement, effec- } \\
\text { tuer les opérations } \\
\text { d'urgence }\end{array}$ & $\begin{array}{l}\text { Indicateurs techniques } \\
\text { issus d'opérations } \\
\text { manuelles ou automa- } \\
\text { tiques. Informations } \\
\text { ponctuelles et syn- } \\
\text { thèses par secteur. } \\
\text { Indicateurs de perfor- } \\
\text { mance spatialisés }\end{array}$ & Oui & $G, S, R, A, B$ & Temps réel \\
\hline $\begin{array}{l}\text { Suivi des } \\
\text { usagers }\end{array}$ & $\begin{array}{l}\text { Demande des usagers, } \\
\text { requêtes de factura- } \\
\text { tion, qualité de la pres- } \\
\text { tation. Recherche, } \\
\text { contact, suivi des pros- } \\
\text { pects }\end{array}$ & $\begin{array}{l}\text { Base de donnée usa- } \\
\text { gers avec état des fac- } \\
\text { turations et problèmes, } \\
\text { et des actions com- } \\
\text { merciales. Tableaux de } \\
\text { suivi des usagers }\end{array}$ & Non & $R, A, B$ & $\begin{array}{l}\text { Horizon }=1 \text { jour } \\
\text { Période }= \\
\text { horaire }\end{array}$ \\
\hline $\begin{array}{c}\text { Police des } \\
\text { eaux }\end{array}$ & $\begin{array}{l}\text { Contrôle périodique } \\
\text { sur le terrain du res- } \\
\text { pect des contrats et } \\
\text { des règles : quantités } \\
\text { prélevées, superficies } \\
\text { irriguées. Vérification } \\
\text { des prises d'eau. } \\
\text { Règlement des conflits }\end{array}$ & $\begin{array}{l}\text { Consommations } \\
\text { Surfaces }\end{array}$ & Oui & B & $\begin{array}{l}\text { Horizon }=1 \text { jour } \\
\text { Période }=1 \text { jour }\end{array}$ \\
\hline $\begin{array}{l}\text { Exécution des } \\
\text { opérations de } \\
\text { renforcement } \\
\text { et d'extension }\end{array}$ & $\begin{array}{l}\text { Satisfaire les nouvelles } \\
\text { demandes de } \\
\text { connexion, renforce- } \\
\text { ment ou extension par } \\
\text { la réalisation des tra- } \\
\text { vaux }\end{array}$ & $\begin{array}{l}\text { Relevé et cartographie } \\
\text { des interventions }\end{array}$ & Oui & $R, A, B$ & Temps réel \\
\hline $\begin{array}{l}\text { Mise en place } \\
\text { des contrats }\end{array}$ & $\begin{array}{l}\text { Constituer les dossiers } \\
\text { usagers et réalisation, } \\
\text { transmettre les enga- } \\
\text { gements contractuels, } \\
\text { mettre en service. } \\
\text { Modifier et résilier les } \\
\text { contrats }\end{array}$ & $\begin{array}{l}\text { Base de données des } \\
\text { contrats avec informa- } \\
\text { tions foncières, juri- } \\
\text { diques, techniques }\end{array}$ & Oui & $R, A, B$ & $\begin{array}{l}\text { Horizon }=1 \text { jour } \\
\text { Période }=1 \\
\text { heure }\end{array}$ \\
\hline $\begin{array}{l}\text { Supervision et } \\
\text { contrôle global }\end{array}$ & $\begin{array}{l}\text { Contrôler l'ensemble } \\
\text { des structures de régu- } \\
\text { lation des eaux }\end{array}$ & $\begin{array}{l}\text { Bilan services fournis - } \\
\text { demande, tableaux des } \\
\text { interventions et des } \\
\text { indicateurs de perfor- } \\
\text { mance }\end{array}$ & Oui & $G, S, R$ & $\begin{array}{c}\text { Horizon }=1 \text { jour } \\
\text { Période }=1 \\
\text { heure }\end{array}$ \\
\hline $\begin{array}{l}\text { Gestion et } \\
\text { comptabilité }\end{array}$ & $\begin{array}{l}\text { Assurer la comptabilité } \\
\text { et la gestion adminis- } \\
\text { trative }\end{array}$ & $\begin{array}{l}\text { Pièces comptables et } \\
\text { analytiques }\end{array}$ & Non & $R, A, B$ & $\begin{array}{l}\text { Horizon }=1 \text { jour } \\
\text { Période }=1 \text { jour }\end{array}$ \\
\hline
\end{tabular}

\subsection{Analyse des outils}

Le poste de consultation doit permettre de:

- stocker un grand nombre d'informations,

- structurer l'information,

- gérer des éléments géographiques,

- manipuler des informations descriptives et géographiques (visualisation),

- définir des écrans de dialogue (interface hommel machine).
Le poste de simulation doit permettre de :

- effectuer des calculs rapidement,

- consolider des informations par différents liens,

- visualiser les résultats de simulation.

L'exploitation simultanée d'informations thématiques et géographiques implique la mise en ouvre du SIG. La spécificité d'utilisation nécessite de développer une application particulièrement adaptée aux besoins des utilisateurs. Le tableau 1 résume le contenu du système et les choix retenus par rapport à la demande; il est à rapprocher de la figure $I$. 


\section{RÉSULTATS DE L'ANALYSE EN IRRIGATION ET DRAINAGE}

Au stade de l'analyse où se trouve le groupe de travail AFEID, et après confrontation des diverses expériences en France et à l'étranger, il est dès maintenant possible de présenter le résultat de l'analyse menée dans le cas des produits d'information permettant la prise de décisions :

- stratégiques, correspondant en général à des objectifs à moyen et long terme (planification): c'est l'objet du tableau 2 ;

- logistiques, correspondant plus aux tâches d'opération et maintenance: c'est l'objet du tableau 3.

Ces deux niveaux ont été retenus car ils sont complémentaires, et illustrent bien les approches différentes selon le pas de temps auquel le gestionnaire souhaite disposer de ces produits pour aider à sa décision.

Le tableau 3 montre que toutes les fonctions identifiées à un niveau de décision donné ne nécessitent pas des produits d'information spatialisés, et qu'il serait donc inadéquat de partir des données (spatialisées ou non) pour bâtir un système d'information.

Par ailleurs, on peut remarquer que certaines fonctions, comme celles liées à la maintenance, nécessiteront des produits spatialisés, mais davantage aux échelons tactique et logistique qu'à l'échelon stratégique.

\section{$V$ CONCLUSIONS}

La finalité de cette approche est d'inciter et d'aider les gestionnaires qui souhaitent élargir leur's méthodes de gestion à de nouvelles technologies à:

- analyser leurs besoins en produits d'information plutôt qu'en données ;

- raisonner en processus de décision au-delà des produits d'information :

- raisonner en termes de gestion et d'organisation au-delà des seuls outils ;

- développer un système d'information de type géographique, dédié à leurs besoins, plutôt qu'une base de données trop générale et mal adaptée.

A partir de ces tableaux, il est maintenant possible de définir les données et les types d'applications utilisant des informations spatialisées, et en particulier les données satellitaires. C'est ainsi que le guide CID Télédétection en irrigation et drainage et le guide SIG permettront aux utilisateurs de transposer ces tableaux à leur cas particulier, et de définir ainsi leur système d'information, les données nécessaires à l'élaboration de produits d'information adaptés, et les applications les plus adéquates.

\section{Remerciements}

Les auteurs tiennent à remercier ici les membres du groupe de travail AFEID sur la télédétection et les SIG qui ont participé à la préparation et à la relecture de ce rapport: $\mathrm{P}$. d'Aquino, CIRAD-SAR, F. Axès, SPOT Image, G. Fabre, BRL, P. Garin, Cemagref Irrigation, P. Lagacherie, INRA Science du Sol, P. Le Goulven, ORSTOM Hydrologie, M. Terrazzoni, SCP, P. Triboulet, INRA SAD, et B. Vincent, Cemagref Drainage

\section{BIBLIOGRAPHIE}

11] Agence de l'Eau Adour-Garonne, GEOSYS (1993). Etude de définition et spécification d'un Tableau de bord de la ressource en eau.

[2] Agence de ['Eau Seine-Normandie, CACG (juin 1995). Etude prospective des besoins en eau dans le bassin amont de la Seine-Normandie.

[3] Agence de l'Eau Seine-Normandie, CACG/GEOSYS (1995). - Mise en place d'un système de simulation spatiale et de géo consultation des besoins en eau à l'horizon 2015 , Manuel d'Utilisation et Manuel de Référence.

14] Carlier A. (1992). - Stratégie appliquée à l'audit des Systèmes d'Information, Ed. HERMES.

15] Didier, Bouveryron (1993). - Guide économique et méthodologique des SIG, Ed. HERMES.

[6] Ministère de l'Agriculture et de la Pêche / DERF, GEOSYS (1994). - Définition du plan de mise en cuvre des Systèmes d'Information Géographique (SIG) pour les mesures agri-environnementales dans le domaine de la protection des eaux.

[7] Préfecture de région Midi-Pyrénées. GEOSYS (1993). Proposition d'une stratégie de développement des Systèmes d'Information Géographique (SIG) au sein des services de l'Etat en région.

[8] VIDAL A. (1995). - Remote sensing applied to irrigation, drainage and flood control. Proc. ICID-CID Special Technical Session, Rome (Italy), 13 september 1995. 10 p. (Actes non paginés).

[9] Vidal A., Sagardoy J.A. (1994), - Conclusions of the Workshop on the use of remote sensing techniques in imigation and drainage, Montpellier, France, 2-5 November 1993. GRID Bulletin, 1994 (1), 13-14.

[10] Vidal A., Sagardoy J.A. (1995). - Use of Remote Sensing Techniques in Irrigation and Drainage, Proceedings of the Expert Consultation, Montpellier, France, 2-4 November 1993, FAO Ed., Water Reports n 4 , Rome, 200 p. 